\title{
Demographic Projections: User and Producer Experiences of Adopting a Stochastic Approach
}

\author{
Kim Dunstan ${ }^{1}$ and Christopher Ball ${ }^{2}$
}

\begin{abstract}
Statistics New Zealand is one of the few national statistical agencies to have applied a stochastic (probabilistic) approach to official demographic projections. This article discusses the experience and benefits of adopting this new approach, including the perspective of a key user of projections, the New Zealand Treasury. Our experience is that the change is less difficult to make than might be expected. Uncertainty in the different projection inputs (components) can be modelled simply or with more complexity, and progressively applied to different projection types. This means that not all the different demographic projections an agency produces need to adopt a stochastic approach simultaneously. At the same time, users of the projections are keen to better understand the relative certainty and uncertainty of projected outcomes, given the important uses of projections.
\end{abstract}

Key words: Population; projection; stochastic; uncertainty.

\section{Introduction}

Demographic projections are a high-priority output for most national statistical organisations. They complement other demographic statistics, such as census statistics and population estimates, to give information about possible future changes in the size and composition of populations (and families, households, and the labour force). In doing so, the projections assist planning and decision making in areas such as health, education, housing, retirement planning, and transport.

This article describes the user and producer experiences of adopting a stochastic (probabilistic) approach to official projections of New Zealand's population, ethnic populations, and labour force. It complements the Letter to the Editor of this journal by Bijak et al. (2015) which touches on some related themes. The stochastic approach implemented by Statistics NZ in 2012 was a shift from the conventional deterministic approach used extensively in demographic projections worldwide. Uncertainty and stochastic processes are not exclusive to the population domain, but the future is inherently uncertain, so demographic projections seem an obvious area of application.

This article does not detail the stochastic methods that have been used, or how specific stochastic models are chosen. These are discussed more fully in Dunstan (2011), Woods and Dunstan (2014), and Statistics NZ (2012a, 2012b, 2014, 2015a, 2015b, 2016).

1 Statistics New Zealand, Private Bag 4741, Christchurch 8140, New Zealand. Email: kim.dunstan@stats.govt.nz 2 New Zealand Treasury. BP Box 3724, Wellington 6008, New Zealand. Email: christopher.ball@ treasury.govt.nz 
This process of change offers lessons for others: why change to stochastic projections and how difficult is it to change? Section 2 contrasts the deterministic and stochastic approaches. Section 3 explores the advantages of a stochastic approach to understand why Statistics NZ is one of the few national statistical agencies to have applied a stochastic approach to demographic projections. Section 4 discusses the practical challenges of making the change, while Section 5 presents thoughts on the likely future direction of demographic projections. Section 6 concludes with some recommendations.

\section{Deterministic and Stochastic Approaches Contrasted}

At first glance, Figure 1 suggests little difference between deterministic and stochastic projections of the population. Like most national statistical organisations, Statistics NZ's projections have conventionally been derived deterministically - by combining specific assumptions (e.g., about fertility, mortality, and migration) to produce a single projection. Different projections or scenarios can be produced by systematically combining different assumptions (Figure 1a). Collectively, those different projections can convey something about the relative certainty or uncertainty of different outcomes, but not in any quantified way. Hence, the probability that an outcome will be above or below a given scenario is unknown and is not estimated in a deterministic projection.

Stochastic or probabilistic projections are produced in much the same way as deterministic projections. The most important difference is that the projection assumptions - the critical inputs to the projection model - also include a measure of variability. The stochastic approach typically involves creating multiple simulations for each of the projection assumptions. The simulations vary randomly according to the probability distributions of each assumption derived from empirical models using historical data, or from judgements (Lutz 2009; Booth and Tickle 2008). These simulations of the assumptions are combined in a conventional way, namely using the cohort-component method (Statistics NZ 2012b, 2014, 2015a, 2016), to produce a population simulation or projection.

The input assumptions and resulting population simulations have realistic trajectories, with all the year-to-year fluctuations inherent in the real world. However, the real value of
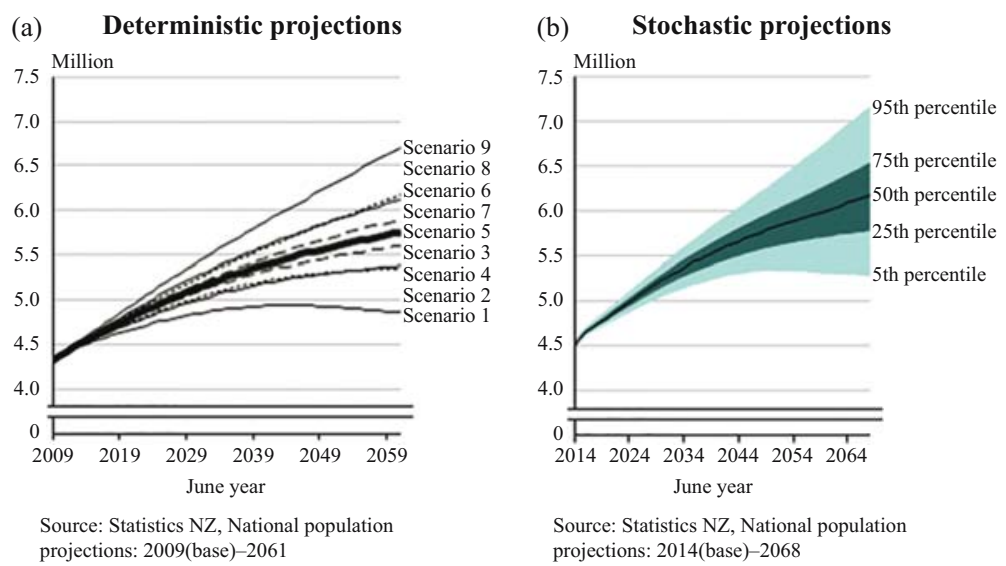

Fig. 1. Alternative projections of the New Zealand population. 
the stochastic approach is not in the individual simulations per se, but in repeating the process and creating many (e.g., 2,000) simulations. From this collective, we can build up a probability distribution of population size and other characteristics. The distribution can be summarised by percentiles, and illustrated using fan charts (Figure 1b), which indicate the probability of different outcomes. In this case, the fifth percentile indicates a five percent chance that the given outcome will be lower than this percentile.

There are many different ways to model uncertainty and to produce stochastic projections. For the purposes of this article, it is sufficient to note that the different approaches share a common aim of conveying uncertainty in a quantified way by allowing the inputs into the models to vary randomly according to probability distributions. Both expectation (e.g., Billari et al. 2014; Lutz et al. 2014; Lutz 2009; Lutz and Scherbov 1998) and empirical approaches are used internationally to model uncertainty, and both have their advantages and disadvantages. The empirical approaches include the propagation of deviations from past projections (e.g., Stoto 1983; Keilman 1997) as well as statistical models (e.g., Wisniowski et al. 2015; United Nations 2014; Raftery et al. 2012), although even these latter approaches include a mix of expert-based inputs. Empirical models have been most intensively applied where demographic trends have been largely monotonic and sustained, as in the case of death rates and life expectancy (e.g., Lee and Carter 1992).

In Statistics NZ's case, statistical models have been developed where possible to generate stochastic measures of the critical projection assumptions, notably for mortality (Woods and Dunstan 2014). However, other assumptions retain the conventional approach of using the expectations (or so-called 'expert judgement') of demographers within Statistics NZ, reflecting that assumptions need to be plausible in both the short term (over the next ten years) and long term (beyond 50 years). Current methods for projections of New Zealand's total population are summarised in Table 1 and highlight that Statistics NZ uses a mix of expectation and empirical approaches. This is a pragmatic approach if not a purely statistical one, but would be the case whether Statistics NZ's projections were deterministic or stochastic.

For projections of ethnic populations - of four broad and overlapping ethnic groups (European, Māori, Asian, and Pacific) - and labour force, a mix of expectation and empirical approaches also applies. These projections include extra assumptions on paternity and interethnic mobility (Statistics NZ 2015a), and labour-force participation and average hours worked (Statistics NZ 2015b). In the case of ethnic projections, greater emphasis is put on expectation due to the shorter historical time series that are available compared with the total population. From a user's perspective, however, this different emphasis makes no visible difference to the projection results.

\section{Benefits of a Stochastic Approach}

The advantages of a stochastic approach are discussed in Alho (1997, 2005), Booth (2006), Bryant (2003, 2005), and Keilman (1991) among others. In this section we raise the discussion above a purely academic or theoretical level by discussing the real benefits experienced in the production and publication of stochastic projections since 2012.

By providing quantification of uncertainty, stochastic projections have assisted the interpretation of projections. There are several aspects to the improved interpretation. 
Table 1. Method of assumption formulation for New Zealand population projections (published 2016).

\begin{tabular}{llc}
\hline Assumption & Median (50th percentile) & Variance/distribution of values \\
\hline $\begin{array}{c}\text { Base } \\
\text { population }\end{array}$ & $\begin{array}{c}\text { Empirical model: official } \\
\text { population estimates based on } \\
\text { census and post-enumeration } \\
\text { survey }\end{array}$ & $\begin{array}{c}\text { Expectation (judgement): } \\
\text { variance varies by age-sex }\end{array}$ \\
Fertility & $\begin{array}{c}\text { Expectation (judgement): } \\
\text { long-term total fertility rate } \\
\text { of 1.85 births per woman }\end{array}$ & $\begin{array}{c}\text { Empirical model: ARIMA } \\
(0,1,0) \text { model fitted to }\end{array}$ \\
& & $\begin{array}{l}\text { total fertility rate for } \\
\text { Empirical model: coherent functional demographic model fitted to }\end{array}$ \\
Mortality & age-specific death rates for 1977-2015 June years \\
& Expectation (judgement): long-term & Empirical model: ARIMA \\
Migration & annual net migration of 15,000 & (1,0,1) model fitted to net \\
& & migration for 1988-2016 \\
& & June years \\
Sex ratio & Empirical model: median and variance from sex ratio at birth for \\
at birth & $1900-2015$ December years & \\
\hline
\end{tabular}

Firstly, the probability distribution of a given variable or characteristic in the stochastic population projections is often skewed, which is typically not conveyed by conventional scenarios. Secondly, the resulting fan charts are intuitive. The 50th percentile or median is analogous to the mid-range deterministic projection that used to be derived. For those people who want one number, they can take that median or indeed another percentile. Finally, the stochastic projections offer more in terms of interpretation. The fan chart of the stochastic projections in Figure 1b, for example, indicates that there is a 50 percent chance that the actual population will lie within the dark band and a 90 percent chance that the actual population will lie within the wider combined lighter and dark band.

By contrast, deterministic scenarios give a poor indication of uncertainty for some key demographic characteristics (e.g., dependency ratios, death numbers). Even for other characteristics, the uncertainty indicated by the scenarios is neither consistent between characteristics, nor consistent across the projection period. For example, Scenarios 1 and 9 give the lowest and highest New Zealand populations, respectively (Figure 1a), but it is Scenarios 3 and 7 which give the lowest and highest ratio of $65+$ population to 15-64 population (Figure 2a). Scenarios 1 and 9 actually give a misleadingly narrow range for the ratio of $65+$ population to 15-64 population. This partly reflects that the 'low' and 'high' variant assumptions combined to give the different scenarios are not equivalent to a consistent probability interval between the fertility, mortality, and migration assumptions (Bongaarts and Bulatao 2000; Bryant 2005; Lee 1998). Deterministic projections can therefore risk giving the impression that a variable or characteristic is more certain than what is presented in stochastic projections.

The use of time-series models is often aimed at improving the accuracy of projections (e.g., United Nations 2014; Woods and Dunstan 2014). However, it is important to clarify 

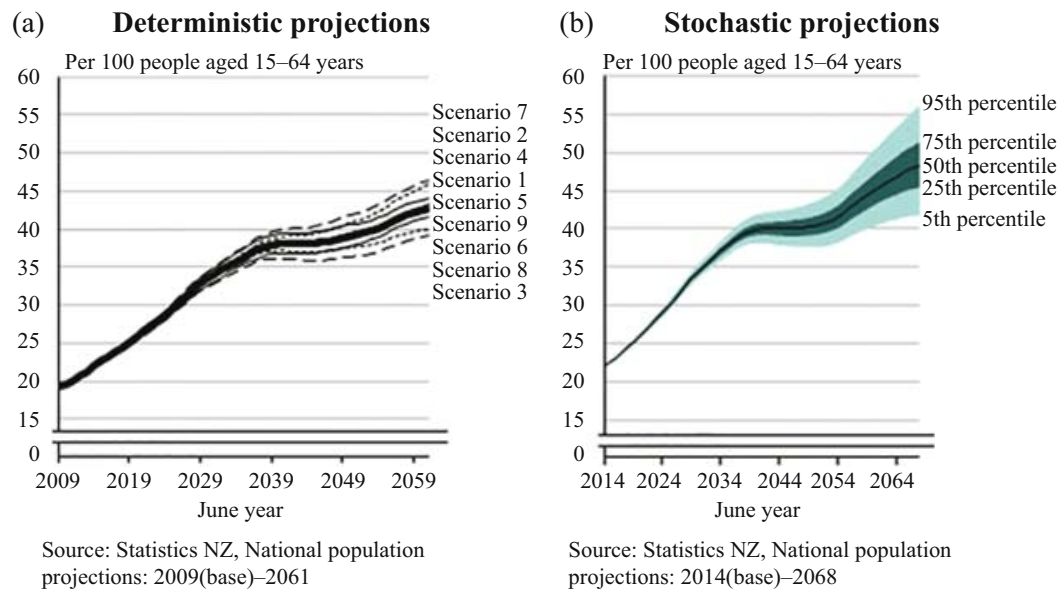

Fig. 2. Alternative projections of the ratio of 65+ population to 15-64 population.

that Statistics NZ's shift to stochastic projections was not driven by an expectation of improving the predictive power or accuracy of the projections. The main driver was to improve the interpretation of projections. Partly, this reflects that Statistics NZ's projections are not produced solely using empirical models (Table 1). Given the inherent uncertainty of the future, encouraging users of projections to think about uncertainty is important. It is only feasible for users to think about uncertainty if that uncertainty is conveyed to them appropriately.

Some other benefits of stochastic projections have yet to be realised, but are worth identifying here as we expect these benefits will be forthcoming. Essentially these benefits are extensions of enhanced interpretation.

First, stochastic projections, with their quantified measures of uncertainty, can actually help statistical agencies make and validate decisions to define the projection period. In principle, there is no limit to how far a projection can be extended (the projection horizon). In practice, statistical agencies are wary of publishing projections with very long horizons, on the basis that the large uncertainty makes the projections more misinformative than informative. For projections of different subpopulations, the horizon might be justifiably different. For example, the stochastic projections for ethnic populations (Statistics NZ 2015a) do have wider uncertainty intervals for all characteristics than the stochastic projections of the total population (Statistics NZ 2014). Moreover, for users of projections, the probability distribution can help them make their own informed decisions about the usefulness of different projections across any projection period.

Second, stochastic projections are generally a better input for users who are involved in modelling and projecting other parameters. For example, stochastic projections help them understand the sensitivity of their projections to the demographic inputs. Or, they help them understand the importance of demographic uncertainty relative to uncertainty coming from, say, economic parameters. For users wanting to use the projections in their own models, they will often need to access the full dataset of simulations. Published percentiles may exclude the specific percentiles needed by specific users, and even a full 
range of percentiles will sometimes be inadequate, especially where users want to extend the stochastic approach to their own models (e.g., with variability estimated for additional parameters).

Population projections are a core input into the New Zealand Treasury's Long Term Fiscal Model (LTFM), which quantifies the sustainability of public finances over a 50year horizon (New Zealand Treasury 2013). Large government expenditure items - such as public pensions, education and health - are sensitive to changes in the age structure of the population, whereas revenue is sensitive to changes in the size of the labour force. The long-term gap between revenue and expenditure, which is typically used to assess fiscal sustainability, is largely driven by changes in the population and labour-force projections.

Before the availability of stochastic population projections, Treasury typically showed sensitivity of the LTFM to the demographic inputs by using deterministic scenarios which altered one of the fertility, mortality, or migration assumptions. These alternative projections gave a range of possible outcomes, but did not give an indication of how likely a given alternative was relative to another projection (including the mid-range projection). Hence, it is difficult for users to test sensitivity to the population inputs by altering single demographic assumptions. Stochastic projections make that assessment explicit and Treasury is now developing the LTFM to incorporate a stochastic approach (Ball et al. 2015).

Stochastic population projections, conveyed using percentiles and fan charts, give users an idea of the mid-range projection, but also place greater emphasis on the uncertainty in each direction. The uncertainty is conditional on past variability (if using empirical approaches) or expert input (if using expectation). More generally, if modelled variability is not indicative of future variability, then the projections may misrepresent the true probability intervals. See Raftery et al. (2012) for an evaluation of calibration of uncertainty, based on probability intervals for 1990-2010 using variability modelled from 1950-1990 data.

The challenges of conveying uncertainty and the nature of the projections generally are new neither from a user perspective nor from a producer perspective and underscore several aspects. First, they emphasise the importance of communicating to users what the projections are (e.g., an indication of future trends based on current policy settings) and how the assumptions have been derived (e.g., how the variability is estimated for each input assumption). Second, it highlights the importance of regularly updating the projections to incorporate changes to levels and variability in the assumptions, as well as to changes in policy settings. Statistics NZ currently updates its projections every $2-3$ years. Third, it reinforces the observation that the value of population projections is only partly defined by whether or not they match reality, especially given the long-term horizon of many projections, but also about whether they are plausible and useful to users at the time the projections were published. Projections aim to form a basis for developing reasonable expectations about the future; to help focus attention on potential events, risks, and opportunities; and to assist people and policy makers to plan and make decisions accordingly. Fourth, this motivates the production of alternative 'what if?' scenarios to complement stochastic projections. These allow particular scenarios of interest to be examined and compared with the benchmark stochastic projections. 


\section{Challenges of Implementing a Stochastic Approach}

National statistical agencies are generally wary of adopting new statistical methodologies. This wariness is warranted, as adopting every new statistical development is neither pragmatic nor cost-effective. In this section we outline the main challenges we faced in adopting a stochastic approach and how these were overcome, as a guide to how other producers of projections might negotiate such challenges.

\subsection{Computing Capacity}

From a production viewpoint, producing large numbers of simulations means that datasets are bigger and programs take longer to run. This can be an issue for users also if they are using those simulations in their own projection models. Computing capacity was a legitimate practical constraint in the past, but it is difficult to justify this as a constraint on implementation in the 21 st century.

\subsection{User Need}

It is sometimes suggested that users of the projections neither want nor need uncertainty to be conveyed in any quantified way. Raftery (2014) identifies five types of general users of projections. Of these, the 'low-stakes user' may have little use for anything but a midrange projection. However, for other user types, an accurate assessment of projection uncertainty is vital as it affects if and how they use the projections.

Importantly, users of projections get nothing less with Statistics NZ's stochastic projections than they got before. They can still get and use one number or projection according to their needs (e.g., median or other percentile). They can still get scenario-type projections with the 'what if?' scenarios which assume fertility, life expectancy, or net migration at specific levels (Statistics NZ 2014, 2015b, 2016). Users need not worry that the projections look radically different to what they did historically. What users also get, however, is better information about uncertainty.

\subsection{User Expectations}

Stochastic projections are not necessarily more accurate (or less accurate) than conventional projections when compared with actuality (i.e., observed population change). There is evidence that empirically based stochastic projections are better than expert-based ones (e.g., Alkema et al. 2011; Raftery et al. 2013). Whether or not they are more accurate, stochastic projections do assist interpretation. Communicating these aspects to users is important to ensure their expectations align realistically with what is produced.

Statistics NZ has not conducted any specific research on the use and understanding of the stochastic projections compared with deterministic projections. However, the development of the stochastic projections from 2005 was gradual rather than a sudden substantial shift in approach. This allowed time for discussions with key users, for discussions at population conferences (e.g., Population Association of New Zealand), for national population prototypes to be developed in 2005 and 2010, and for the publication of a working paper (Dunstan 2011). There was therefore the opportunity for 
both the producer and users to consider the implications of a shift in approach before the new methodology was adopted. Importantly, there has been no negative reaction from users to the adoption of a stochastic approach in the release of official projections since 2012.

\subsection{User Understanding}

Two important aspects of stochastic projections to convey to users is information about the stochastic methods (metadata) and the projections (results) themselves. These aspects are not new for statistical agencies, which have always had the challenge of conveying technical aspects and detailed data to a variety of users.

The experience of Statistics NZ was that existing products and services were suitable for disseminating the stochastic projections. Conventional information releases presented summary results via commentary, graphs and tables (Statistics NZ 2012a, 2012b, 2014, 2015a, 2015b, 2016). More detailed data for each of the projections were disseminated using the existing web-based tool NZ.Stat, a table-builder product powered by software provided by the OECD (Statistics NZ 2012c, OECD 2013).

Conventionally, projections of different characteristics from different scenarios would be published. With the stochastic projections, different percentiles (5th, 10th, 25th, 50th, 75th, 90th, and 95th) of those same characteristics are published. These published percentiles appear to have satisfied user demand, although other percentiles can be readily supplied on request. Given the trend of increasing data dissemination, one can envisage users in future being able to select any percentiles of interest to them, or even the individual simulations for use in their own modelling.

In the two years following the publication of the first stochastic population projections in 2012, the number of unique visitors viewing the main NZ.Stat table (population by agesex) online averaged more than 100 per month. We are not aware of any of those users requiring assistance to interpret the projections through the website feedback forms or Statistics NZ's free helpline. While this could simply indicate that users are not seeking assistance when they need it, the continued use and downloading of the stochastic projections seems to suggest that users generally understand them.

\subsection{Spurious Precision}

The estimates of uncertainty are themselves uncertain and Statistics NZ has always been upfront about this (Statistics NZ 2012a, 2012b, 2014, 2015a, 2015b, 2016). The projections do not try to include uncertainty arising from catastrophic events (e.g., earthquakes, wars). As they are projections based on current policy settings, they do not try to anticipate major policy changes, so there is the additional uncertainty of 'nondemographic' factors (assuming these factors are not captured in the modelling of 'demographic' uncertainty). The estimates of uncertainty depend on what historical data is used and how uncertainty is modelled. While it is possible to estimate uncertainty based on the historical variability of the demographic parameters, it is more difficult to estimate the uncertainty that arises from the choice of models (for one approach, see Abel et al. 2013), or from the choice of time period(s) that affect the model parameters. 
Dowd et al. (2010) refer to these three different types of uncertainty as:

1. model uncertainty (e.g., we do not know the true fertility model),

2. parameter uncertainty (e.g., whatever mortality model we use, we do not know the true values of its parameters),

3. projection uncertainty (e.g., the uncertainty of future migration rates given any particular model and its calibration).

There may be additional uncertainty arising from factors such as errors in historical data and errors in expert judgement (see Keilman 1991; Alho 1997; De Beer 2000). Notably, Bayesian methods are capable of combining various sources of uncertainty in a coherent manner, including, for example, expert uncertainty, model uncertainty, parametric uncertainty, and covariate uncertainty (see Bijak and Bryant 2016). In effect, many stochastic projections (including those of Statistics NZ) model only the projection uncertainty, and are therefore inclined to underestimate the true uncertainty. Nonetheless, this is an improvement on conventional deterministic projections which do not model any of the types of uncertainty. So while the estimated probability intervals in the stochastic projections may appear spuriously precise, this is preferable to a spuriously precise deterministic projection.

One of the challenges for Treasury in moving to a stochastic projection framework is conveying a more accurate assessment of the fiscal pressures without giving the impression of spurious accuracy. In fact, stochastic projections better allow users to focus on broad trends, without overemphasising small changes in the mid-range projection that occur between updates every few years. But there is a challenge in conveying the full uncertainty in fiscal projections in addition to the uncertainty coming from the demographic projections. There are judgements around the effects of government policy and economic variables which are also subject to uncertainty. As a result, the current fiscal projections underestimate the true range of uncertainty.

\subsection{Impact on Other Projections}

Like many statistical agencies, Statistics NZ produces a suite of demographic projections, including projections of subpopulations (Figure 3). These projections are not produced

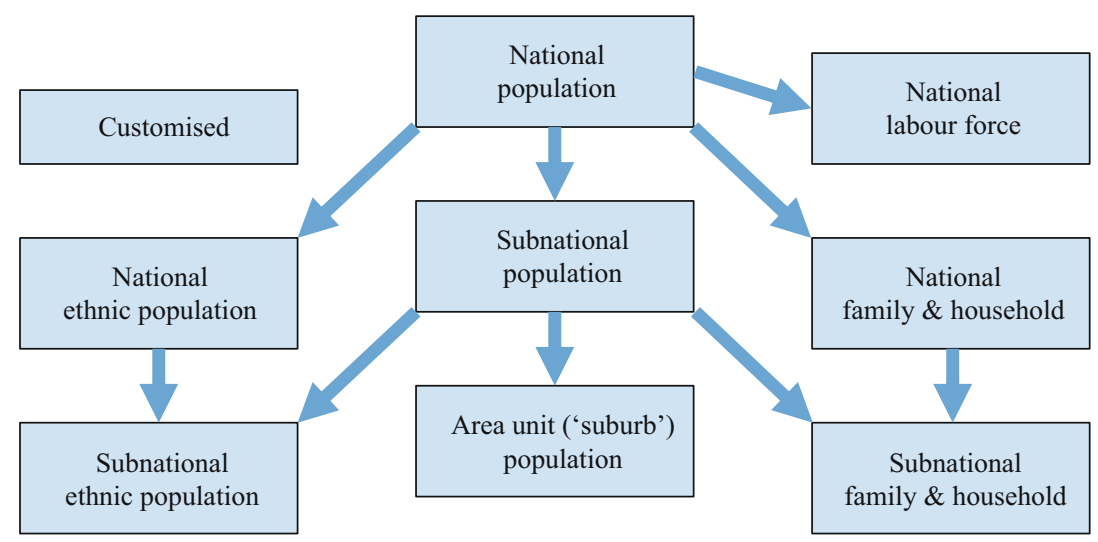

Fig. 3. Demographic projections produced by Statistics NZ. 
using a single model (e.g., micro-simulation), but are produced using independent projection models, albeit designed explicitly or implicitly to produce an internally consistent set of projections. For example, the mid-range projection for subnational areas will align with the mid-range national projection. This additivity of geographical areas is highly sought by users and contributes to the general coherence of the projections produced.

One of the attractions of Statistics NZ's stochastic approach is that it can be applied to one or more of its projection types without compromising the other projections. For example, a stochastic approach was applied to national population and labour-force projections in 2012, and was extended to national ethnic population projections in 2015. Other projections, including all subnational projections, have continued in their more conventional deterministic form. This is an important consideration for statistical agencies. who might see the challenge of producing stochastic projections for subpopulations a barrier to producing any stochastic projections at all. Long and Hollman (2004) discuss this in relation to ethnic and Hispanic population projections in the United States.

\subsection{Cost}

A detailed assessment of the costs to develop and produce stochastic projections is beyond the scope of this article, but a few observations based on Statistics NZ's experience are pertinent. First, there are nontrivial investment costs to formulate measures of uncertainty using empirical and/or expectation approaches, to embed the stochastic methods within the organisational production process, and to engage with users in advance of adoption. However, these are initial one-off costs and producers of projections can potentially benefit from the development of stochastic projections by the United Nations, Statistics NZ and elsewhere, thereby reducing the cost and time of their own development. The availability of free open-source software (e.g., Sevcikova et al. 2011; Hyndman 2015) also means cost need not be an obvious deterrent to implementation.

Beyond the development phase, the ongoing production costs have been similar to those of producing conventional deterministic projections. Additional resources are required to formulate measures of uncertainty and to produce multiple simulations when each set of projections is produced, although this is offset by not needing to produce alternative deterministic assumptions (e.g., 'low' and 'high' variants). It was possible to use existing products and services to disseminate metadata and the stochastic projections.

\section{Future Developments}

In common with other statistical agencies, Statistics NZ has an expressed set of strategic objectives to which it aspires. These include being a trusted provider of official statistics, empowering customers in data understanding and use, and driving value for customers through the use of innovative tools and techniques (Statistics NZ 2015c). The development of stochastic projections can be viewed as concomitant with the organisation's core values and strategic direction. As a producer of official (population) statistics, high priority is placed upon the statistical rigour of data, concepts, and methods, including incorporating 
international best practices. However, there is also an onus on Statistics NZ to provide statistical leadership (e.g., in statistical methodologies), which can run counter to more conservative statistical practices.

Lutz and Scherbov (1998) discuss how "the change of a long-established tradition" generally requires the following:

1. The new practice must have clear advantages when compared with the current one.

2. It should be consistent with other work done by the producing institution, and present an evolution along established lines rather than a discontinuity.

3. The proposed approach should be internally consistent and based on accepted scientific work.

4. It should be practical for both the users and producers, and not cost too much.

What is the experience of Statistics NZ in terms of these criteria? Stochastic projections do offer clear advantages, as discussed in this article. The adoption of a stochastic approach has been a progressive evolution in methodology and, we would argue, not a paradigm shift. For example, the traditional cohort-component method remains the basis of the projections, but with the addition of a stochastic dimension. The stochastic approach builds on a large body of published work from respected demographers and statisticians worldwide. The publication of stochastic projections (Statistics NZ 2012a, 2012b, 2014, 2015a, 2015b, 2016; United Nations 2014) shows that they are practical to produce, and once developed the production costs are similar to conventional methods.

These are important messages for other producers of projections, or for users of projections looking to influence producers of projections. Others can leverage off stochastic developments in New Zealand and elsewhere. Our experience shows that a stochastic approach can be applied to selected projection types while maintaining consistency with other projections produced deterministically. Projection assumptions can be formulated using time series or other empirical models and/or using expert-based approaches. In addition, Statistics NZ continues to publish hypothetical 'what if?' scenarios which are useful in illustrating the effect of specific fertility, mortality, and migration assumptions on population size and structure. A blend of deterministic and stochastic methods is therefore a pragmatic approach for producers of projections, and useful from a user's perspective.

Statistics NZ is aiming to apply stochastic approaches to its other demographic projections - of families and households, and of subnational populations - with the common rationale of increasing the interpretability and usefulness of the projections. Extending the stochastic approach is not without further challenges, and may require different modelling approaches to that used already, such as the use of Bayesian modelling for subnational projections (Bryant and Graham 2013; Bijak and Bryant 2016).

We should expect to see more countries and agencies producing stochastic projections in future. Partly this will be driven by users of projections wanting more informative indications of future demographic change, given the importance of projections for planning and decision making. Partly this will be driven by practical solutions, assisted by technology, to overcome the challenges of adopting a stochastic approach.

More generally, we can expect a growing integration of statistical methods into demographic projections (and other applications) in New Zealand and internationally. This collaboration should be encouraged by producers and users of projections, as the 
combination of statistical knowhow with demographic knowledge should strengthen the value of projections. In particular, users can have increased confidence in projections which have a strong statistical and data-driven basis, yet remain plausible and interpretable. The development of global stochastic population projections by the United Nations Population Division is likely to add momentum to their uptake.

\section{Conclusion}

From a user's perspective, stochastic projections have been a welcome development. Conventional deterministic projections were poor at conveying the inherent uncertainty of future changes. By quantifying uncertainty, stochastic projections assist interpretation. They clarify which demographic trends are probable and which are improbable. By giving users some quantification of the probability of an event occurring, they can make the case for intervention much sharper. Importantly, there has been no negative reaction from users to the adoption of a stochastic approach in the release of official projections since 2012.

In addition to these benefits, the stochastic projections are inspiring users to quantify additional components of uncertainty. For example, Ball et al. (2015) explore uncertainty in economic parameters such as interest rates, productivity growth, and government expenditure. Such work might be expected to flow through to products dependent on demographic projections, such as long-term fiscal projections, to fully convey the distribution of uncertainty.

The following recommendations provide guidance for producers of projections considering adopting a stochastic approach:

1. Engage with users of projections. Do they understand and use alternative deterministic projections, such as 'low' and 'high' variants? Would they benefit from more informed measures of uncertainty in projections?

2. Identify institutional barriers to adopting a stochastic approach.

3. Look for opportunities to collaborate with other organisations, researchers and academics on using and developing stochastic methods.

4. Utilise existing open-source software to produce stochastic projections in testing and productions.

5. Utilise published examples of how stochastic projections are disseminated in terms of metadata (explanatory information) and data (projection results).

6. Consider producing 'what if?' deterministic scenarios to complement the principal stochastic projections to illustrate specific demographic scenarios.

7. Consider applying a stochastic approach to selected projection types (e.g., national population) before extending further. A progressive development allows projection types to be tackled in order of complexity, and can also help manage user expectations.

From a producer's perspective, stochastic projections are meeting a user need. Increasingly, producers are more focussed on measuring and conveying uncertainty, and less on conveying overly precise point estimates. There are few practical obstacles to producing stochastic population projections, other than the additional resources required to formulate measures of uncertainty and produce multiple simulations. Moreover, given 
the nature of projections, a stochastic approach is consistent with how statistical agencies would like projections to be conveyed and interpreted.

\section{References}

Abel, G., J. Bijak, J. Forster, J. Raymer, P. Smith, and J. Wong. 2013. "Integrating Uncertainty in Time Series Population Forecasts: An Illustration Using a Simple Projection Model.” Demographic Research 29: 1187-1226. Doi: http://dx.doi.org/10. 4054/DemRes.2013.29.43.

Alho, J. 1997. "Scenarios, Uncertainty and Conditional Forecasts of the World Population." Journal of the Royal Statistical Society: Series A (Statistics in Society) 160: 71-85. Doi: http://dx.doi.org/10.1111/1467-985X.00046.

Alho, J. 2005. "Remarks on the Use of Probabilities in Demography and Forecasting." In Perspectives on Mortality Forecasting: Probabilistic Models, edited by N. Keilman. Social Insurance Studies No. 2, Swedish Social Insurance Agency. Available at: www. forsakringskassan.se/wps/wcm/connect/95496cc1-ada3-41a6-af4c-02b4b0b3c233/ social_insurance_studies_2.pdf?MOD=AJPERES (accessed 19 May 2016).

Alkema, L., A. Raftery, P. Gerland, S. Clark, F. Pelletier, T. Buettner, and G. Heilig. 2011. "Probabilistic Projections of the Total Fertility Rate for All Countries." Demography 48: 815-839. Doi: http://dx.doi.org/10.1007/s13524-011-0040-5.

Ball, C., J. Creedy, and G. Scobie. 2015. Long-Run Fiscal Projections Under Uncertainty: The Case of New Zealand. New Zealand Treasury Working Paper 15/10. Available at: www.treasury.govt.nz (accessed 19 May 2016).

Bijak, J., I. Alberts, J. Alho, J. Bryant, T. Buettner, J. Falkingham, J. Forster, P. Gerland, T. King, L. Onorante, N. Keilman, A. O’Hagan, D. Owens, A. Raftery, H. Sevcikova, and P. Smith. 2015. "Letter to the Editor: Probabilistic Population Forecasts for Informed Decision Making.” Journal of Official Statistics 31: 537-544. Doi: http://dx.doi.org/10. 1515/jos-2015-0033.

Bijak, J. and J. Bryant. 2016. "Bayesian Demography 250 Years after Bayes.” Population Studies 70: 1-19. Doi: http://dx.doi.org/10.1080/00324728.2015.1122826.

Billari, F., R. Graziani, and E. Melilli. 2014. "Stochastic Population Forecasting Based on Combinations of Expert Evaluations Within the Bayesian Paradigm.” Demography 51: 1933-1954. Doi: http://dx.doi.org/10.1007/s13524-014-0318-5.

Bongaarts, J. and R. Bulatao. 2000. Beyond Six Billion: Forecasting the World's Population. Washington, DC: National Academy Press. Doi: http://dx.doi.org/10.17226/9828.

Booth, H. 2006. "Demographic Forecasting: 1980 to 2005 in Review." International Journal of Forecasting 22: 547-581. Doi: http://dx.doi.org/10.1016/j.ijforecast.2006. 04.001.

Booth, H. and L. Tickle. 2008. "Mortality Modelling and Forecasting: a Review of Methods." Annals of Actuarial Science 3: 3-43. Doi: http://dx.doi.org/10.1017/ S1748499500000440.

Bryant, J. 2003. "Can Population Projections Be Used for Sensitivity Tests on Policy Models?" New Zealand Treasury Working Paper 03/07. Available at: www.treasury. govt.nz/publications/research-policy/wp/2003/03-07 (accessed 19 May 2016). 
Bryant, J. 2005. "What Can Stochastic Population Projections Contribute to Policy Analysis?" New Zealand Population Review 31: 1-11. Available at: www.population. org.nz/wp-content/uploads/2010/01/nzpr-vol-31-1_bryant.pdf (accessed 19 May 2016).

Bryant, J. and P. Graham. 2013. "Bayesian Demographic Accounts: Subnational Population Estimation Using Multiple Data Sources." Bayesian Analysis 8: 591-622. Doi: http://dx.doi.org/10.1214/13-BA820.

De Beer, J. 2000. "Dealing with Uncertainty in Population Forecasting". Statistics Netherlands. Available at: www.cbs.nl/nr/rdonlyres/7dc466f9-fe4c-48dc-be9030216b697548/0/dealingwithuncertainty.pdf (accessed 19 May 2016).

Dowd, K., D. Blake, and A. Cairns. 2010. "Facing Up to Uncertain Life Expectancy: The Longevity Fan." Demography 47: 67-78. Doi: http://dx.doi.org/10.1353/dem.0.0083.

Dunstan, K. 2011. "Experimental Stochastic Population Projections for New Zealand: 2009(base)-2111." Statistics New Zealand Working Paper 11-01. Available at: http:// www.stats.govt.nz/methods/research-papers/working-papers-original/experimentalstochastic-pop-projections-nz-11-01.aspx (accessed 19 May 2016).

Hyndman, R. 2015. "Demography." Available at: http://robjhyndman.com/software/ demography/ (accessed 19 May 2016).

Keilman, N. 1991. "Uncertainty in National Population Forecasting: Issues, Backgrounds, Analyses, Recommendations." International Journal of Forecasting 7: 392-393. Doi: http://dx.doi.org/10.1016/0169-2070(91)90018-Q.

Keilman, N. 1997. "Ex-Post Errors in Official Population Forecasts in Industrialized Countries." Journal of Official Statistics 13: 245-277. Available at: www.jos.nu/ Articles/abstract.asp?article=133245 (accessed 19 May 2016).

Lee, R. 1998. "Probabilistic Approaches to Population Forecasting." In Frontiers of Population Forecasting, edited by W. Lutz, J. Vaupel, and D. Ahlburg. 156-190. New York: The Population Council.

Lee, R. and L. Carter. 1992. "Modeling and Forecasting US Mortality." Journal of the American Statistical Association 87: 659-671. Doi: http://dx.doi.org/10.2307/2808055.

Long, J. and F. Hollman. 2004. "Developing Official Stochastic Population Forecasts at the US Census Bureau." International Statistical Review 72: 201-208. Available at: www.jstor.org/stable/1403854 (accessed 19 May 2016).

Lutz, W. 2009. Toward a Systematic, Argument-Based Approach to Defining Assumptions for Population Projections. Interim Report IR-09-037, International Institute for Applied Systems Analysis, Austria. Available at: http://pure.iiasa.ac.at/9115/1/IR-09-037.pdf (accessed 19 May 2016).

Lutz, W., W. Butz, and S. KC. 2014. World Population and Human Capital in the 21st Century. Oxford University Press. Doi: http://dx.doi.org/10.1111/j.1728-4457.2015. 00032.x.

Lutz, W. and S. Scherbov. 1998. “An Expert-Based Framework for Probabilistic National Population Projections: The Example of Austria." European Journal of Population 14: 1-17. Doi: http://dx.doi.org/10.1023/A:1006040321755.

New Zealand Treasury. 2013. “Affording Our Future: Statement on New Zealand's LongTerm Fiscal Position." Available at: http://www.treasury.govt.nz/government/ longterm/fiscalposition/2013/affordingourfuture (accessed 19 May 2016). 
OECD. 2013. “OECD.Stat Web Browser User Guide”. Available at: https://stats.oecd.org/ Content/themes/OECD/static/help/WBOS\%20User\%20Guide\%20(EN) (accessed 25 October 2016).

Raftery, A. 2014. "Use and Communication of Probabilistic Forecasts." Available at: http://arxiv.org/abs/1408.4812 (accessed 19 May 2016).

Raftery, A., J. Chunn, P. Gerland, and H. Sevcikova. 2013. "Bayesian Probabilistic Projections of Life Expectancy for All Countries.” Demography 50: 777-801. Doi: http://dx.doi.org/10.1007/s13524-012-0193-x.

Raftery, A., N. Li, H. Sevcikova, P. Gerland, and G. Heilig. 2012. "Bayesian Probabilistic Population Projections for All Countries." In Proceedings of the National Academy of Sciences 109(35): 13915-13921. Available at: www.pnas.org/content/109/35/13915. full.pdf + html (accessed 19 May 2016).

Sevcikova, H., L. Alkema, and A. Raftery. 2011. "bayesTFR: An R Package for Probabilistic Projections of the Total Fertility Rate." Journal of Statistical Software 43: 1-29. Doi: http://dx.doi.org/10.18637/jss.v043.i01.

Statistics NZ. 2012a. "National Labour Force Projections: 2006(base)-2061 (August 2012 update)." Available at: http://www.stats.govt.nz/browse_for_stats/population/ estimates_and_projections/NationalLabourForceProjections_HOTP06-61Augupdate. aspx (accessed 19 May 2016).

Statistics NZ. 2012b. "National Population Projections: 2011(base)-2061.2." Available at: http://www.stats.govt.nz/browse_for_stats/population/estimates_and_projections/ NationalPopulationProjections_HOTP2011.aspx (accessed 19 May 2016).

Statistics NZ. 2012c. "NZ.Stat user guide.” Available at: http://nzdotstat.stats.govt.nz/ wbos/ModalHelp/OECD/WBOS\%20User\%20Guide\%20(EN).PDF (accessed 19 May 2016).

Statistics NZ. 2014. "National Population Projections: 2014(base)-2068." Available at: http://www.stats.govt.nz/browse_for_stats/population/estimates_and_projections/ NationalPopulationProjections_HOTP2014.aspx (accessed 19 May 2016).

Statistics NZ. 2015a. "National Ethnic Population Projections: 2013(base)-2038." Available at: http://www.stats.govt.nz/browse_for_stats/population/estimates_and_ projections/NationalEthnicPopulationProjections_HOTP2013-38.aspx (accessed 19 May 2016).

Statistics NZ. 2015b. "National Labour Force Projections: 2015(base)-2068”. Available at: http://www.stats.govt.nz/browse_for_stats/population/estimates_and_projections/ NationalLabourForceProjections_HOTP15-68.aspx (accessed 19 May 2016).

Statistics NZ. 2015c. "Statement of Strategic Intentions for Statistics New Zealand 2015-19.” Available at: http://www.stats.govt.nz/about_us/what-we-do/our-publications/ statement-of-intent/statement-of-intent-2015.aspx (accessed 25 October 2016).

Statistics NZ. 2016. "National Population Projections: 2016(base)-2068." Available at: http://www.stats.govt.nz/browse_for_stats/population/estimates_and_projections/ NationalPopulationProjections_HOTP2016.aspx (accessed 25 October 2016).

Stoto, M. 1983. "The Accuracy of Population Projections." Journal of the American Statistical Association 78: 13-20. Doi: http://dx.doi.org/10.1080/01621459.1983. 10477916. 
United Nations. 2014. "World Population Prospects: The 2012 Revision, Methodology of the United Nations Population Estimates and Projections." Working Paper No. ESA/P/Wp. 235. Available at: https://esa.un.org/unpd/wpp/publications/Files/ WPP2012_Methodology.pdf (accessed 25 October 2016).

Wisniowski, A., P. Smith, J. Bijak, J. Raymer, and J. Forster. 2015. "Bayesian Population Forecasting: Extending the Lee-Carter Method." Demography 53: 1035-1059. Doi: http://dx.doi.org/10.1007/s13524-015-0389-y.

Woods, C. and K. Dunstan. 2014. "Forecasting Mortality in New Zealand: A New Approach for Population Projections Using a Coherent Functional Demographic Model." (Statistics New Zealand Working Paper No 14-01). Available at: http://www. stats.govt.nz/methods/research-papers/working-papers-original/forecasting-mortality14-01.aspx (accessed 19 May 2016).

Received May 2015

Revised June 2016

Accepted August 2016 